INVESTIGACIÓN

Recibido: 11/07/2018 --- Aceptado: 29/04/2019 --- Publicado: 15/09/2019

\title{
NOCIÓN DE LA EDUCOMUNICACIÓN EN LAS ORGANIZACIONES INTERGUBERNAMENTALES
}

\section{The notion of the educommunication in intergovernmental organizations}

Mari Carmen Caldeiro Pedreira1. Universidad Santiago de Compostela. España. mcarmen.caldeiro@usc.es

Ángel Torres-Toukoumidis. Universidad Politécnica Salesiana. Ecuador. atorrest@ups.edu.ec

Luis M. Romero-Rodríguez. Universidad Rey Juan Carlos. España. luis.romero@urjc.es

Ignacio Aguaded. Universidad de Huelva. España. ignacio@aguaded.es

\section{RESUMEN}

La presente investigación analiza la noción que tienen las principales organizaciones internacionales de carácter multilateral sobre el concepto de educomunicación (media literacy), mediante el extracto correlacional y análisis de contenido de base interpretativa de 2.648 unidades informativas de 12 organismos, tanto de carácter regional como transcontinental, entre los que se encuentran la Organización de las Naciones Unidas, Organización para la Cooperación y el Desarrollo Económico, Liga Árabe, Organización Internacional de la Francofonía, Comunidad de Países de Lengua Portuguesa y Organización de Estados Iberoamericanos, Unión Africana, Unión Europea, Liga de los Estados Árabes, Comunidad de Estados Independientes, Organización de Estados Americanos, Comunidad de Estados Latinoamericanos y Caribeños, Unión de Naciones Sudamericanas, Asociación de Naciones del Sureste Asiático, Consejo de Cooperación para los Estados Árabes del Golfo y Foro de las Islas del Pacífico. En cuanto a los principales resultados se evidencia que $83 \%$ organizaciones analizadas, salvo en la Comunidad de Países de Lengua Portuguesa y en la Unión Africana la convergencia del

\footnotetext{
${ }^{1}$ Mari Carmen Caldeiro Pedreira: Doctora en Comunicación y Educación por la Universidad de Huelva (España). Máster en Formación del Profesorado por la Universidad de Santiago de Compostela (España). Profesora de Pedagogía y Didáctica de la Universidad de Santiago de Compostela (España).
} 
Caldeiro Pedreira, M.; Torres-Toukoumidis, A., Romero-Rodríguez, L. M. y Aguaded, I. Noción de la educomunicación en las organizaciones intergubernamentales

contexto educativo y comunicativo, específicamente se presenta bajo la terminología "educación mediática", lo que influye en la formulación de políticas domésticas mediante el planteamiento de incentivos y restricciones que afectan la toma de decisiones a nivel gubernamental aludiendo al continuo cambio y la dinámica construcción de la identidad.

PALABRAS CLAVE: alfabetización mediática - organizaciones intergubernamentales análisis de contenido - medios digitales - TIC - educomunicación - gobierno.

\section{ABSTRACT}

This research analyzes the notion that the main international organizations of multilateral character have on the concept of educommunication (media literacy), by means of the correlational extract and analysis of content of interpretative base of 2.648 information units from 12 organizations, both regional and transcontinental, including the United Nations, the Organization for Economic Cooperation and Development, the Arab League, the International Organization of la Francophonie, the Community of Portuguese-speaking Countries and the Organization of Ibero-American States, African Union, European Union, League of Arab States, Commonwealth of Independent States, Organization of American States, Commonwealth of Latin American and Caribbean States, Union of South American Nations, Association of Southeast Asian Nations, Cooperation Council for the Arab States of the Gulf and Pacific Islands Forum. Regarding the main results, it is evident that $83 \%$ of the organizations analyzed, except in the Community of Portuguese Language Countries and in the African Union, the convergence of the educational and communicative context, is specifically presented under the terminology "media education", which influences the formulation of domestic policies through the proposal of incentives and restrictions that affect decision making at the governmental level, alluding to the continuous change and the dynamic construction of identity.

KEY WORDS: media literacy - intergovernmental organizations - content analysis digital media - ICT - educommunication - government.

\section{NOÇÃO DA EDUCOMUNICAÇÃO NAS ORGANIZAÇOES INTER-GOVERNAMENTAIS}

\section{RESUME}

A presente investigação analisa a noção que tem as principais organizações internacionais de caráter multilateral sobre o conceito de educomunicação (media literacy), mediante o estrato de correlação e analise de conteúdo de base interpretativa de 2648 unidades informativas de doze organismos, tanto de caráter regional como transcontinental, entre os que se encontram a O.N.U., Organização para a Cooperação e

Vivat Academia. Revista de Comunicación. 15 septiembre 2019 /15 diciembre 2019, n 148, 23-40 
Caldeiro Pedreira, M.; Torres-Toukoumidis, A., Romero-Rodríguez, L. M. y Aguaded, I. Noción de la educomunicación en las organizaciones intergubernamentales

o Desenvolvimento Econômico, Liga Árabe, Organização Internacional da Francófona, Comunidade de países de língua portuguesa e Organização de estados iberoamericanos, Union Africana, Union Europeia, Liga dos Estados Árabes, Comunidade de Estados Independentes, Organização de Estados Americanos, Comunidade de Estados Latino-americanos e Caribenhos, União de Nações Sul-americanas, Associação de Nações do Sudeste Asiático, Conselho de Cooperação dos Estados Árabes do Golfo e Fórum das Ilhas do Pacifico. Enquanto aos principais resultados evidencia-se que 83 por cento das organizações analisadas salvo na Comunidade de Países de Língua Portuguesa e na União Africana a convergência do contexto educativo e comunicativo, especificamente se apresenta sobe a terminologia "Educação mediática", o que influi na formulação de políticas domésticas mediante a proposta de incentivos e restrições que afetam a toma de decisões a nível governamental aludindo à continua mudança e a dinâmica construção da identidade-

PALAVRAS CHAVE: alfabetização mediática - organizações intergovernamentais analises de conteúdo - meios digitais - TIC - educomunicação - governo.

\section{Como citar el artículo:}

Caldeiro Pedreira, M.; Torres-Toukoumidis, A., Romero-Rodríguez, L. M. y Aguaded, I. (2019). Noción de la educomunicación en las organizaciones intergubernamentales. [The notion of the educommunication in intergovernmental organizations]. Vivat Academia. Revista de Comunicación, 148, 23-40.

DOI: http:// doi.org/10.15178/va.2019.148.23-40

Recuperado de http://www.vivatacademia.net/index.php/vivat/article/view/1127

\section{INTRODUCCIÓN}

El contexto hipermedia en el que vivimos condiciona no solo las relaciones humanas sino además la formación y capacitación de los sujetos. Tanto la educación formal como la no formal o la informal, exenta esta última de una actividad organizada, sistemática y que se desarrolla fuera del sistema educativo, contribuyen al desarrollo de la identidad de la ciudadanía.

El hecho de que se institucionalice el uso de nuevos dispositivos tecnológicos como modo de comunicación o que las conexiones a Internet se realicen de manera imparable a escala mundial, determina la conformación de la identidad de la población usuaria de estos medios.

En cierta medida, asistimos a un momento en el cual la "infoxicación" y la "infopolución" se convierten en baluartes de una sociedad cada vez más digitalizada (Aguaded y Romero-Rodríguez, 2015). La identidad digital, el uso y consumo de las

Vivat Academia. Revista de Comunicación. 15 septiembre 2019 /15 diciembre 2019, nº 148, 23-40 
Caldeiro Pedreira, M.; Torres-Toukoumidis, A., Romero-Rodríguez, L. M. y Aguaded, I. Noción de la educomunicación en las organizaciones intergubernamentales

herramientas y dispositivos móviles o la convergencia casi total de lo audiovisual y lo digital justifican el análisis de la realidad educomunicativa actual y su relación con la conformación de la identidad del sujeto (Romero-Rodríguez, De-Casas-Moreno y Torres-Toukoumidis, 2016). Actualmente no es suficiente consumir medios y contenidos audiovisuales, sino que resulta imprescindible el desarrollo de la competencia mediática que favorece el alcance del prosumer (Sánchez y Sandoval-Romero, 2012) o como indican Hernández, Renés y Greenhill $(2017$, p. 77$)$ produser, es decir, nuevas audiencias que componen el ecosistema mediático actual y que son capaces de no solo recibir sino además producir contenidos de forma mínimamente crítica y responsable. Una producción que se desarrolla en un ambiente en el cual impera la conectividad permanente y que requiere necesariamente de la capacitación crítica del receptor, sea cual fuere su edad (Caldeiro y Aguaded, 2015, p. 39). Una empresa que se atribuye de manera aleatoria y en ocasiones poco racional al ámbito educativo si bien conforma parte de la labor de diferentes agentes sociales como pueden ser, entre otros, los propios medios o las familias. Según Bauman (2000) los hombres y mujeres de la modernidad son el resultado de las innovaciones digitales y la comprensión de contenidos audiovisuales. Esta comprensión puede entenderse como el fruto de un proceso de maduración en el cual la pedagogía cumple un papel determinante. En definitiva y de forma general se persigue el empoderamiento y desarrollo de la mirada crítica que permite la decodificación de los múltiples lenguajes existentes.

Por un lado y para contribuir a ésta se propone la educación de sujetos autónomos y críticos que centra el interés de la investigación surgida en torno a la consecución de personas que cultivan la capacidad crítica. Esta habilidad debe forjarse en sociedad y ha de implementarse desde la institución educativa, de tal forma que trasciende los meros espacios académicos en los cuales tiempo atrás únicamente se aprendían contenidos relacionados con las diferentes disciplinas.

En el contexto académico donde se han producido -especialmente a lo largo de los últimos 20 años- reformas educativas, se demanda la inclusión en el currículo de contenidos transversales que constituyan un eje vertical y global en la capacitación de la ciudadanía. Estos contenidos requieren de la integración curricular de las tecnologías interactivas (Gutiérrez-Martín, 2007). Una exigencia que se acentúa especialmente cuando el desarrollo tecnológico y el contexto mediático actual exigen la incorporación, en el aula de las fuentes de información con las que conviven los adolescentes en su día a día. En este sentido, y con el fin de alcanzar los objetivos indicados, se sugiere la alfabetización que persigue la elaboración colaborativa de la mirada crítica (Uribe, 2010). Una producción que corresponde a la tarea educomunicativa, que se focaliza en el aprendizaje producido en un ambiente mediático donde predomina la comunicación en la que intervienen no solo los emisores sino además activamente los receptores.

Vivat Academia. Revista de Comunicación. 15 septiembre 2019 /15 diciembre 2019, nº 148, 23-40 
Caldeiro Pedreira, M.; Torres-Toukoumidis, A., Romero-Rodríguez, L. M. y Aguaded, I. Noción de la educomunicación en las organizaciones intergubernamentales

$\mathrm{Al}$ esfuerzo de las diferentes instituciones educativas se suma la tarea de los agentes sociales que conforman la mirada del receptor. En este entramado, y para su alcance, desempeña un papel determinante el empoderamiento que favorece la decodificación de los múltiples lenguajes existentes. Para ello resulta fundamental el binomio comunicación-educación que se convierte en núcleo fundamental de la cultura TIC, en la que se encuentran inmersas las nuevas generaciones.

\section{OBJETIVOS}

La identidad del prosumer se construye en la sociedad hipermedia de la mano de diferentes agentes que comparten un objetivo común: el alcance de sujetos no solo críticos y autónomos sino que desarrollen la habilidad que les permite analizar y producir contenidos de forma responsable.

Una tarea que compete, entre otros y como se ha indicado, a los entornos escolares y familiares. A lo largo de la década de los setenta, diferentes instituciones de carácter supraestatal como la propia UNESCO han demandado la formación transversal en materia educomunicativa y su inclusión en el currículo académico. De igual modo el Parlamento Europeo, a través de su programa de media literacy "UNAOC" buscaba la elaboración de productos audiovisuales por jóvenes de todo el mundo (Aguaded, 2011). En este sentido se han desarrollado diferentes experiencias, especialmente en Europa; prácticas como el foro de Media Literacy que se ha desarrollado en 2010 en Reino Unido o el congreso nacional de Literacia, Media e Cidadania celebrado en Portugal en 2011.

En España, pese a que se han celebrado diferentes congresos, simposios y foros sobre esta temática, continúa siendo una tarea pendiente para el alcance de la cual aúnan esfuerzos entre otros, el Grupo Comunicar de Huelva o el Gabinete de Comunicación dirigido por José Manuel Pérez-Tornero en la Universidad Autónoma de Barcelona. Unos y otros trabajan en pro de la educomunicación, un término que ha sido explicado desde diferentes vertientes por múltiples expertos y asociaciones sensibilizadas con la formación para el desarrollo de la "ciudadanía mediática" (Gozálvez, 2013). Con el fin de explicar el término han surgido diferentes aproximaciones como las que pueden observarse en la siguiente tabla:

Tabla 1. Principales aproximaciones teóricas al concepto de educomunicación.

\begin{tabular}{|l|l|}
\hline $\begin{array}{l}\text { Conferencia UNESCO } \\
\text { sobre Educomunicación } \\
(1982)\end{array}$ & $\begin{array}{l}\text { Forma de crear las capacidades para tener una relación } \\
\text { autónoma, ponderada y crítica con todos los medios, a partir } \\
\text { de conceptos como libertad de expresión, libertad de } \\
\text { información, ética, entre otros. }\end{array}$ \\
\hline Kaplún (1997) & $\begin{array}{l}\text { La educomunicación es una forma de involucrar en el proceso } \\
\text { de interacción a los diferentes miembros de la sociedad. }\end{array}$ \\
\hline García-Matilla (2002) & La educomunicación aspira a dotar a toda persona de las \\
\hline
\end{tabular}

Vivat Academia. Revista de Comunicación. 15 septiembre 2019 /15 diciembre 2019, nº 148, 23-40 
Caldeiro Pedreira, M.; Torres-Toukoumidis, A., Romero-Rodríguez, L. M. y Aguaded, I. Noción de la educomunicación en las organizaciones intergubernamentales

\begin{tabular}{|l|l|}
\hline & $\begin{array}{l}\text { competencias expresivas imprescindibles para su normal } \\
\text { desenvolvimiento comunicativo y para el desarrollo de su } \\
\text { creatividad. }\end{array}$ \\
\hline Buckingham (2003) & $\begin{array}{l}\text { La educomunicación es la incorporación de la acción } \\
\text { productiva por parte del sujeto, admitiendo al mismo tiempo } \\
\text { la lectura y la escritura, la interpretación o recepción y la } \\
\text { producción mediática, introduciendo a su vez elementos } \\
\text { icónicos, digitales y audiovisuales. }\end{array}$ \\
\hline Aparici (2010) & $\begin{array}{l}\text { La educomunicación se la conoce también como la recepción } \\
\text { crítica de los medios de comunicación. }\end{array}$ \\
\hline Pérez-Tornero y Varis & $\begin{array}{l}\text { La educomunicación es el proceso de asumir y ysar los códigos } \\
\text { implicados en el sistema mediática contemporáneo, así como } \\
\text { las habilidades prácticas necesarias para un uso adecuado de } \\
\text { los sistemas tecnológicos en que estos códigos se basan. }\end{array}$ \\
\hline Aguaded (2012) & $\begin{array}{l}\text { La educomunicación es una formación cultural que tiene como } \\
\text { objeto potenciar la integración de la cultura mediática en las } \\
\text { escuelas, la transformación de los medios de comunicación } \\
\text { hacia un objeto de estudio y análisis curricular, y la } \\
\text { transferencia de conocimiento en la vida cotidiana. }\end{array}$ \\
\hline
\end{tabular}

Fuente: Elaboración propia.

La educomunicación persigue la integración de los medios y las tecnologías como parte de la totalidad de la vida del sujeto, tanto de los menores como de los adultos. Acostumbra a identificarse con la educación mediática que favorece el alcance e implantación de la alfabetización de la ciudadanía basada en el desarrollo de habilidades críticas que implica tender un puente entre expresión oral, impresión, imagen, imagen en movimiento y alfabetización en medios digitales (Tyner, 2008).

En la forja de este concepto, la formación a lo largo de la vida o el manejo de los múltiples dispositivos tecnológicos y la comprensión de los contenidos audiovisuales trascienden la divergencia de quienes tildan a los medios de elementos que disgregan frente a quienes les consideran nexos de unión que fomentan el contacto intercultural y social.

Durante la primera década del actual siglo y últimos años del pasado han ido surgiendo trabajos que si bien conforman un marco teórico encomiable, adolecen de estrategias que impelen al lector al menos a preocuparse por la necesidad de realizar análisis de índole práctico. El corpus teórico se sustenta sobre informes acerca de la situación y análisis que, más allá de una apología de la realidad, sitúa entre sus principales pretensiones la realización de actividades de carácter transversal que pueden materializarse en la práctica totalidad de asignaturas que componen el currículo.

Todo ello conlleva la necesidad de "pensar en modelos de aprendizaje continuos" (Cobo y Movarec 2011, p. 19) que sientan la base del diseño de propuestas para la

Vivat Academia. Revista de Comunicación. 15 septiembre 2019 /15 diciembre 2019, nº 148, 23-40 
Caldeiro Pedreira, M.; Torres-Toukoumidis, A., Romero-Rodríguez, L. M. y Aguaded, I. Noción de la educomunicación en las organizaciones intergubernamentales

formación de la identidad. Estas matrices formativas trascienden los contenidos curriculares e incluso conforman el currículo oculto del aula y al mismo tiempo demandan la inclusión de nuevas estrategias, certificando la necesidad de repensar la educación e incluyendo el aprendizaje no reglado, al que diversos autores denominan "aprendizaje invisible"; aprendizaje que no solo integra distintas ideas y perspectivas, sino que persigue el desarrollo del capital humano y además puede producirse en cualquier lugar y momento, casi de forma omnipresente. Asimismo, trasciende la mera formalidad para referirse al aprendizaje no formal, a fin de construir conexiones que permitan estrechar la relación entre conceptos (Cobo y Movarec, 2011, p. 25).

De manera genérica se justifica la forja de conocimientos en la sociedad tecnológica que exige la alfabetización digital y el desarrollo de las competencias favorables a la resolución de problemas originados en contextos donde predominan las tecnologías. Se trata de contextos en los cuales es necesaria la interpretación y comprensión de la información (Ralón, Vieta y Vásquez, 2004).

En este contexto se justifica el fomento del autoaprendizaje y el desarrollo de estrategias que permitan, no solo el manejo sino, además, el entendimiento y significado de los medios y la información que transmiten. Para ello debemos contar con un sistema diferente al que proponía la "educación bancaria" que apelaba a la memorización y archivo de contenidos y que generan sujetos acríticos (Freire, 1984). Para paliar tal carencia se propone, entre otras formas, el aprendizaje basado en proyectos que incluye la integración de la tecnología y apuesta por el diseño y la creatividad como pilares básicos y competencias principales en el siglo XXI. Una forma de trabajo esta última que justifica el trabajo colaborativo con base en el diálogo, la comprensión y la elaboración de materiales e ideas adaptadas a la situación para la cual han sido creadas. Se trata de desarrollar propuestas orientadas a vincular múltiples espacios donde pueda fomentar el aprendizaje a través de las diferentes fuentes de trasmisión de ideología, costumbres y valores.

\section{METODOLOGÍA}

La presente investigación se enfoca en analizar las diferentes aproximaciones y corrientes teóricas al concepto que otorgan las organizaciones internacionales de carácter multilateral (regionales y transcontinentales) sobre la educomunicación (media literacy) dentro de un contexto global. Para alcanzar el precitado objetivo, se propone revisar de forma sistemática el enfoque al término "educomunicación" en las páginas web oficiales de las siguientes organizaciones transcontinentales: Organización de las Naciones Unidas, Organización para la Cooperación y el Desarrollo Económico, Liga Árabe, Organización Internacional de la Francofonía, Comunidad de Países de Lengua Portuguesa y Organización de Estados Iberoamericanos. De igual modo, se incluyen dentro de la muestra de análisis las organizaciones internacionales de carácter regional:

Vivat Academia. Revista de Comunicación. 15 septiembre 2019 /15 diciembre 2019, nº 148, 23-40 
Caldeiro Pedreira, M.; Torres-Toukoumidis, A., Romero-Rodríguez, L. M. y Aguaded, I. Noción de la educomunicación en las organizaciones intergubernamentales

Unión Africana, Unión Europea, Liga de los Estados Árabes, Comunidad de Estados Independientes, Organización de Estados Americanos, Comunidad de Estados Latinoamericanos y Caribeños, Unión de Naciones Sudamericanas, Asociación de Naciones del Sureste Asiático, Consejo de Cooperación para los Estados Árabes del Golfo y Foro de las Islas del Pacífico.

La estrategia de búsqueda aplicada se dirige principalmente a la combinación de palabras en inglés en motores de búsquedas generales bajo limitación semántica exclusiva (algoritmo “..."), de ese modo, la información de los resultados se concentra en las frases que mantienen el mismo orden de las palabras y excluye aquellos resultados que no tienen vinculación exacta con los términos. Asimismo, se tradujeron las tres palabras clave "educación mediática", "alfabetización mediática" y "competencias mediáticas" de la búsqueda al idioma oficial o principal de cada organización, tal como se muestra en la siguiente tabla:

Tabla 2. Características de la estrategia de búsqueda.

\begin{tabular}{|c|c|c|c|c|}
\hline Organización & $\begin{array}{c}\text { Idioma } \\
\text { principal }\end{array}$ & Palabra clave 1 & Palabra clave 2 & Palabra clave 3 \\
\hline Naciones Unidas & Inglés & "media education" & "media literacy" & $\begin{array}{c}\text { "media } \\
\text { competences" }\end{array}$ \\
\hline $\begin{array}{l}\text { Organización para } \\
\text { la Cooperación y el } \\
\text { Desarrollo } \\
\text { Económico }\end{array}$ & Inglés & "media education" & "media literacy" & $\begin{array}{c}\text { "media } \\
\text { competences" }\end{array}$ \\
\hline $\begin{array}{l}\text { Liga de los Estados } \\
\text { Árabe }\end{array}$ & Árabe & ةبيمالعإلا ةيبرتلا & ةبيمالعإلا ةفاقثل| & تئماءافكالكل| \\
\hline $\begin{array}{l}\text { Organización } \\
\text { Internacional de la } \\
\text { Francofonía }\end{array}$ & Francés & $\begin{array}{l}\text { "éducation aux } \\
\text { médias" }\end{array}$ & $\begin{array}{l}\text { "alphabétisation } \\
\text { médiatique" }\end{array}$ & $\begin{array}{l}\text { "compétence } \\
\text { médiatique" }\end{array}$ \\
\hline $\begin{array}{l}\text { Comunidad de } \\
\text { Países de Lengua } \\
\text { Portuguesa }\end{array}$ & Portugués & $\begin{array}{l}\text { "educação de } \\
\text { mídia" }\end{array}$ & $\begin{array}{l}\text { "literacia } \\
\text { mediática" }\end{array}$ & $\begin{array}{l}\text { "competências } \\
\text { de mídia" }\end{array}$ \\
\hline $\begin{array}{l}\text { Organización de } \\
\text { Estados } \\
\text { Iberoamericanos }\end{array}$ & Español & $\begin{array}{l}\text { "educación } \\
\text { mediática" }\end{array}$ & $\begin{array}{l}\text { "alfabetización } \\
\text { mediática" }\end{array}$ & $\begin{array}{l}\text { "competencias } \\
\text { mediáticas" }\end{array}$ \\
\hline Unión Africana & Inglés & "media education" & "media literacy" & $\begin{array}{c}\text { "media } \\
\text { competences" }\end{array}$ \\
\hline Unión Europea & Inglés & "media education" & "media literacy" & $\begin{array}{c}\text { "media } \\
\text { competences" }\end{array}$ \\
\hline $\begin{array}{l}\text { Comunidad de } \\
\text { Estados } \\
\text { Independientes }\end{array}$ & Ruso & медиаобразование & медиаграмотности & $\begin{array}{c}\text { медиа- } \\
\text { компетенции }\end{array}$ \\
\hline $\begin{array}{l}\text { Organización de } \\
\text { Estados } \\
\text { Americanos }\end{array}$ & Inglés & "media education" & "media literacy" & $\begin{array}{c}\text { "media } \\
\text { competences" }\end{array}$ \\
\hline Asociación de & Inglés & "media education" & "media literacy" & "media \\
\hline
\end{tabular}

Vivat Academia. Revista de Comunicación. 15 septiembre 2019 /15 diciembre 2019, n 148, 23-40 
Caldeiro Pedreira, M.; Torres-Toukoumidis, A., Romero-Rodríguez, L. M. y Aguaded, I. Noción de la educomunicación en las organizaciones intergubernamentales

\begin{tabular}{|l|c|c|c|c|}
\hline \multicolumn{1}{|c|}{ Organización } & $\begin{array}{c}\text { Idioma } \\
\text { principal }\end{array}$ & Palabra clave 1 & Palabra clave 2 & Palabra clave 3 \\
\hline $\begin{array}{l}\text { Naciones del } \\
\text { Sureste Asiático }\end{array}$ & & & & competences" \\
\hline $\begin{array}{l}\text { Foro de las Islas del } \\
\text { Pacífico }\end{array}$ & Inglés & "media education" & "media literacy" & $\begin{array}{c}\text { "media } \\
\text { competences" }\end{array}$ \\
\hline
\end{tabular}

Fuente: Elaboración propia.

Por su parte, el segundo objetivo específico se orienta a sintetizar la información oficial extraída de 12 organizaciones intergubernamentales relacionadas con la educomunicación. En este apartado se explica la posición de referencia en cada una de las organizaciones consultadas, asumiendo los múltiples propósitos de su implementación a través de los criterios expuestos en el marco teórico:

- Convergencia del contexto educativo y comunicativo: Avalar la convergencia entre educación y comunicación desarrollando competencias específicas que potencien el pensamiento crítico.

- Configuración dentro de contexto educativo: Otorgar predominancia a los aspectos vinculados a la educación y al aprendizaje.

- Configuración dentro de un contexto comunicativo: Otorgar predominancia a los aspectos de la comunicación y de la transmisión informativa.

Para alcanzar los objetivos citados ut supra, se plantea un diseño de investigación de carácter cualitativo bajo extracto correlacional, utilizando una metodología de análisis de contenido de base interpretativa, entendido como aquel que "involve interpretation of findings across studies to generate new inductive understandings of the phenomena, events or experiences" (Saini \& Shlonsky, 2012, p. 31). El uso de este método se justifica porque la muestra se configura meramente por fuentes documentales de las que se extraerán, mediante procesos inductivos, correlaciones semánticas y conceptos de índole teóricos, omitiendo la interacción del investigador en el proceso de recolección de datos (Sampieri, Collado y Lucio, 2014), teniendo como norte la comprensión del fenómeno central del estudio, conociendo los antecedentes, situaciones producidas y funcionamiento (Lecompte y Schensul, 2012).

\section{RESULTADOS}

Del procedimiento de búsqueda citado en referencia emergieron un total de 2.648 unidades informativas, que conforman la muestra efectiva (Me), entre las cuales se incluyen documentos como noticias, comunicados oficiales, reportes, conferencias, proyectos, actividades, entre otros. Igualmente cabe destacar que de las 12 organizaciones intergubernamentales sometidas a la revisión exhaustiva de su página web oficial con respecto a las tres palabras clave en sus idiomas principales, solamente dos de ellas (Comunidad de Países de Lengua Portuguesa, Unión Africana) no tienen

Vivat Academia. Revista de Comunicación. 15 septiembre 2019 /15 diciembre 2019, nº 148, 23-40 
Caldeiro Pedreira, M.; Torres-Toukoumidis, A., Romero-Rodríguez, L. M. y Aguaded, I. Noción de la educomunicación en las organizaciones intergubernamentales

información vinculada a la educomunicación, pese a tener en su organigrama un departamento, comisión o comité relacionado con la educación.

Tabla 3. Resultados emergentes de palabras clave por organización.

\begin{tabular}{|l|c|c|c|}
\hline \multicolumn{1}{|c|}{ Organización } & $\begin{array}{c}\text { "educación } \\
\text { mediática" }\end{array}$ & $\begin{array}{c}\text { "alfabetización } \\
\text { mediática" }\end{array}$ & $\begin{array}{c}\text { "competencias } \\
\text { mediáticas" }\end{array}$ \\
\hline Naciones Unidas & 206 & 88 & 6 \\
\hline $\begin{array}{l}\text { Organización para la Cooperación y el } \\
\text { Desarrollo Económico }\end{array}$ & 110 & 86 & 3 \\
\hline Liga Árabe & 17 & 38 & 0 \\
\hline $\begin{array}{l}\text { Organización Internacional de la } \\
\text { Francofonía }\end{array}$ & 3 & 0 & 0 \\
\hline $\begin{array}{l}\text { Comunidad de Países de Lengua } \\
\text { Portuguesa }\end{array}$ & 0 & 0 & 0 \\
\hline Organización de Estados Iberoamericanos & 40 & 82 & 2 \\
\hline Unión Africana & 0 & 0 & 0 \\
\hline Unión Europea & 220 & 1.467 & 10 \\
\hline Comunidad de Estados Independientes & 0 & 0 & 0 \\
\hline Organización de Estados Americanos & 37 & 23 & 0 \\
\hline $\begin{array}{l}\text { Asociación de Naciones del Sureste } \\
\text { Asiático }\end{array}$ & 8 & 1 & 0 \\
\hline Foro de las Islas del Pacífico & 3 & 0 & 219 \\
\hline Sub-total & 644 & 1.785 & \\
\hline Total & & $\mathbf{2 . 6 4 8}$ & \\
\hline
\end{tabular}

Fuente: Elaboración propia.

\subsection{Organización de las Naciones Unidas (ONU)}

Las Naciones Unidas consideran la alfabetización mediática como una de las prioridades transversales en la educación actual, ya que impulsa la promoción del acceso equitativo a la información y posibilita la evaluación crítica de la calidad del contenido transmitido. Desde esta visión, la estrategia consiste en asociarle con la alfabetización informacional, la correlación de ambas disciplinas complementa la experiencia formativa equipando al ciudadano con las competencias básicas necesarias para actuar frente a los retos del siglo XXI.

Si bien la UNESCO -entidad adscrita a la ONU- demuestra la incorporación de otras disciplinas en el desarrollo de la educomunicación, la misma se realiza desde una visión multidisciplinaria, es decir, se mantienen separados los campos de estudio, omitiendo el entrecruzamiento de variables entre las disciplinas que la conforman. En cambio, se evidencia claramente el criterio orientado a la convergencia entre la educación y

Vivat Academia. Revista de Comunicación. 15 septiembre 2019 /15 diciembre 2019, nº 148, 23-40 
Caldeiro Pedreira, M.; Torres-Toukoumidis, A., Romero-Rodríguez, L. M. y Aguaded, I. Noción de la educomunicación en las organizaciones intergubernamentales

comunicación, reiterando continuamente el empoderamiento de las personas mediante la formación de un pensamiento crítico y aprehensión de competencias que permitan socializar y convertirse en ciudadanos activos.

\subsection{Organización para la Cooperación y el Desarrollo Económico (OCDE)}

Las informaciones extraídas tienden a una aproximación empírica donde se observan múltiples estudios de caso sobre la aplicación de la educomunicación. La línea de análisis abarca principalmente la configuración dentro de contexto educativo, profundizando en cuanto a la implementación, infraestructura, interacción, formación docente y creación de programas estratégicos de las Tecnologías de Comunicación e Información (TIC). En complemento, esta organización posee un Centro para la investigación educativa e Innovación (CERI- Centre for Educational Research and Innovation) donde dos de los seis proyectos actuales están vinculados estrechamente con la educomunicación, a saber: i) Education and Social Progress, ii) Open Higher Education and Innovative Learning Environments. Al fin y al cabo, se puede dilucidar la influencia de las nuevas tecnologías, la formación digital y la interconexión informática en la educación, como el fundamento conceptual que esta organización internacional tiene sobre la educomunicación.

\subsection{Liga Árabe}

Mediante un acuerdo de cooperación con el departamento de documentación e información, la institución encargada de liderar los proyectos educativos de la Liga Árabe se denomina Arab League Educational, Cultural and Scientific Organization (ALECSO) enfocándose específicamente en la educación para adultos y en el desarrollo de la cultura. La educomunicación se concibe con la finalidad de salvaguardar la identidad y simbolismo del lenguaje arábigo y la unidad de su área geográfica de competencia. En efecto, manteniendo la misma línea de la Organización de las Naciones Unidas, se inclina al desarrollo de actividades educativas y comunicativas que fortalecen las capacidades de análisis crítico de los mensajes transmitidos por los medios de comunicación.

\subsection{Organización Internacional de la Francofonía}

La idea general exhibe la predisposición para la creación de proyectos orientados a la utilización responsable, al desarrollo de buenas prácticas y la fomentación de incentivo de participación a través de los medios. Esto significa que en esta organización, el eje conductor de la educomunicación es la configuración dentro de un contexto comunicativo, protagonizado por los soportes, medios, canales y contenido emitido.

\subsection{Comunidad de Países de Lengua Portuguesa}

Si bien queda reflejado ut supra que esta organización carece de unidades informativas emergentes relacionadas con la educomunicación, de la misma manera se

Vivat Academia. Revista de Comunicación. 15 septiembre 2019 / 15 diciembre 2019, nº 148, 23-40 
Caldeiro Pedreira, M.; Torres-Toukoumidis, A., Romero-Rodríguez, L. M. y Aguaded, I. Noción de la educomunicación en las organizaciones intergubernamentales

pudo constatar que en el acta constitutiva de Lisboa 1996, se persigue el criterio teórico de la configuración dentro del contexto educativo buscando la optimización de los sistemas educativos a través de recursos provenientes de las nuevas tecnologías de información y comunicación, desarrollo de la enseñanza a distancia y profesionalización del ciclo medio.

\subsection{Organización de Estados Iberoamericanos (OEI)}

Esta organización cuenta con dos estrategias relacionadas con la difusión de educomunicación. La primera de ellas, seleccionar manuscritos de revista científicas y memorias de congresos con acceso libre orientándose a la temática (Sánchez y SandovalRomero, 2012) y, en segundo lugar, la realización de un boletín de novedades y noticias sobre tecnología y comunicación educativa, poniéndose en evidencia una propensión por la conciencia y actitud crítica hacia los medios. De allí que se priorice como criterio teórico la convergencia entre en contexto educativo y comunicativo.

\subsection{Unión Africana (UA)}

En la noción de un conteniente próspero, pacífico e integrado no circunda en la incorporación explícita de la educomunicación. Pese a ello, se observan comisiones como: "asuntos sociales" y "sociedad civil y diáspora" en los que asumen la importancia de la educación para lograr efectos positivos duraderos. Igualmente, en la agenda 2063 se hace mención al concepto de educación para la paz, la cual según Davies (2005) propone soluciones al igual que la educación mediática para luchar contra una visión dogmática de la sociedad. Adicionalmente, Sterling y Huckle (2014), manifiestan que el establecimiento de ambas constituye las bases la educación sostenible.

\subsection{Unión Europea (UE)}

La información presentada por la Unión Europea supera con creces aquella publicada tanto por sus homólogos regionales como de sus pares intergubernamentales. Esto implica que la educomunicación está escenificada como un concepto arraigado en la cultura europea. De hecho, la Comisión Europea (2008), reafirmando las bases de la Declaración de Grünwald sobre Educación Mediática (1982), esgrime que el objetivo de los programas de alfabetización en medios es la promoción activa y consciente de la ciudadanía europea. Más aún, se reivindica la convergencia entre educación y comunicación mediante la necesidad de aprender a evaluar críticamente el contenido de los medios de comunicación, y adquirir capacidades vinculadas a la creatividad como la curiosidad, la toma de riesgos y la resolución de problemas, condicionando así en gran medida la confianza de los usuarios hacia las tecnologías digitales y, por tanto, la adopción de las TIC. De hecho, en el programa de investigación e innovación Horizon 2020, se propone el tema: Media Literacy in the Digital Environment, para desarrollar

Vivat Academia. Revista de Comunicación. 15 septiembre 2019 / 15 diciembre 2019, nº 148, 23-40 
Caldeiro Pedreira, M.; Torres-Toukoumidis, A., Romero-Rodríguez, L. M. y Aguaded, I. Noción de la educomunicación en las organizaciones intergubernamentales

nuevas metodologías, herramientas y evidencia científica en contextos digitales para el beneficio de gobiernos, empresas e individuos.

\subsection{Comunidad de Estados Independientes}

La información emitida por esta organización precisó un filtrado detallado sobre los aspectos que corresponden a la educomunicación, ya que existe eco ideológico dentro de la temática que desvía la investigación académica. No obstante, la palabra clave "competencias mediáticas" en ruso, permitió sintetizarlo en la convergencia entre la educación y comunicación a través del aprendizaje de un proceso de lectura especializado en textos incorporando el análisis crítico, evaluación, creación y transferencia de habilidades.

\subsection{Organización de Estados Americanos (OEA)}

Según la visión de esta organización, la educomunicación se emplea como vehículo para la participación democrática, donde compatibiliza la educación cívica, las tecnologías de comunicación e información y los espacios de transmisión audiovisual. En otras palabras, la convergencia comunicación-educación se formaliza por acuerdos de resolución como la 1885 (XXXII-O/02) o el reporte anual de la Comisión de los Derechos Humanos (2015), documentos en los que se sugiere la configuración de campañas "educomunicativas" sobre planes de contingencia orientados a la estimulación de la perspectiva crítica y al libre acceso de las nuevas tecnologías.

\subsection{Asociación de Naciones del Sureste Asiático}

En el directorio de Desarrollo Humano se encuentra la división denominada Educación, Juventud y Deportes. Esta entidad se encarga de emitir reportes anuales y memorias de eventos de distinta índole, donde se evidencia la envergadura de la "alfabetización mediática". De hecho, trabajan esta temática desde 1991 cuando la UNESCO le proporcionó recursos para diseñar material didáctico adaptado a la realidad mediática. En efecto, desde esta organización se observa cierta continuidad del colectivo hacia el desarrollo de la "educomunicación" y su configuración dentro de contexto educativo.

\subsection{Foro de las Islas del Pacífico}

Más allá de exploraciones protocolares que demuestran un rol dentro en la configuración dentro del contexto educativo, anunciando futuros planes de estudio y desarrollo de la educomunicación, se establecen problematizaciones de mayor urgencia dentro del ámbito educativo, entre ellas el deterioro de la infraestructura y la deserción escolar.

Vivat Academia. Revista de Comunicación. 15 septiembre 2019 /15 diciembre 2019, nº 148, 23-40 
Caldeiro Pedreira, M.; Torres-Toukoumidis, A., Romero-Rodríguez, L. M. y Aguaded, I. Noción de la educomunicación en las organizaciones intergubernamentales

\section{DISCUSIÓN}

Las instituciones intergubernamentales influyen en la formulación de políticas domésticas de cada país mediante el planteamiento de incentivos y restricciones que afectan la toma de decisiones a nivel gubernamental (Heinmiller, 2007). Por tanto, reconociendo que la muestra seleccionada abarca organizaciones transcontinentales y regionales, consta que la educomunicación, específicamente vista bajo el término "educación mediática", tiene presencia en las noticias, comunicados oficiales, reportes, conferencias, proyectos y actividades en el $83 \%$ organizaciones analizadas, salvo en la Comunidad de Países de Lengua Portuguesa y en la Unión Africana.

En efecto, la información de las organizaciones consultadas demuestra que la tendencia mayoritaria dentro de la educomunicación es la convergencia del contexto educativo y comunicativo. Es decir, la Organización de las Naciones Unidas, la Liga Árabe, la Organización de Estados Iberoamericanos, la Unión Europea, la Organización de Estados Americanos y la Comunidad de Estados Independientes buscan la integración del usuario en la sociedad, la filiación cultural y la visión crítica hacia el contenido de los medios de comunicación. En segundo lugar, la Organización para la Cooperación y el Desarrollo Económico, la Asociación de Naciones del Sureste Asiático y el Foro de las Islas del Pacífico se encaminan hacia el contexto educativo proponiendo formación docente, diseño de material didáctico y anunciando futuros planes de estudio que incorporen la educomunicación. En tercera y última instancia, la Organización Internacional de la Francofonía es la única organización dentro de la muestra que manifiesta de manera explícita sus políticas de educomunicación, priorizando la configuración de contexto comunicativo, motivando el desarrollo de buenas prácticas y conocimiento especializado de los soportes, medios y canales de la comunicación. En definitiva, las recientes formas de comunicación requieren de un mayor grado de formación y las nuevas necesidades precisan de una serie de cambios y adaptaciones a la situación emergente, por ello, la educomunicación, formalizada en este estudio como "educación mediática" no es más que el resultado de un proceso que se nutre de la comprensión e interiorización de los diferentes rasgos característicos del sujeto que vive en sociedad, aludiendo al continuo cambio y la dinámica construcción de la identidad.

Para futuros trabajos, se sugiere profundizar sobre las experiencias en educomunicación de los países que conforman las organizaciones intergubernamentales consultadas, de tal modo que se pueda tener una visión comparativa sobre las políticas domésticas con respecto a los programas de las diversas organizaciones de adscripción. Asimismo, según Chibás (2012) los ecosistemas formados a través de la educomunicación lo constituyen cuatro dimensiones: educación, comunicación, gestión estratégica y de proyectos, y creatividad. Si bien en este estudio se toman en cuenta dos de ellos, en próximas investigaciones se deberían incluir los dos restantes para ampliar el panorama teórico de la educomunicación.

Vivat Academia. Revista de Comunicación. 15 septiembre 2019 /15 diciembre 2019, nº 148, 23-40 
Caldeiro Pedreira, M.; Torres-Toukoumidis, A., Romero-Rodríguez, L. M. y Aguaded, I. Noción de la educomunicación en las organizaciones intergubernamentales

\section{REFERENCIAS}

Aguaded, I., y Romero-Rodríguez, L. M. (2015). Mediamorfosis y desinformación en la infoesfera: Alfabetización mediática, digital e informacional ante los cambios de hábitos de consumo informativo/Mediamorphosis and misinformation in the infosphere: media, digital and information literacy face of changes in information consumption habits. Education in the Knowledge Society, 16(1), 44-59. DOI: https://doi.org/10.14201/eks20151614457

Aguaded, I. (2012). La educomunicación: una apuesta de mañana, necesaria para hoy. Aularia: Revista Digital de Comunicación, 1(2), 259-261.

Aguaded-Gómez, I. (2011). La educación mediática, un movimiento internacional imparable. La ONU, Europa y España apuestan por la educomunicación. Comunicar, 19(37), 7-8. DOI: https://doi.org/10.3916/c37-2011-01-01

Aparici, R. (2011). Educomunicación: más allá del 2.0. Barcelona, España: Editorial Gedisa.

Bauman, Z. (2015). Modernidad líquida. Buenos Aires, Argentina: Fondo de cultura económica.

Buckingham, D. (2013). Media education: Literacy, learning and contemporary culture. Cambridge, Inglaterra: John Wiley \& Sons.

Caldeiro-Pedreira, M. C., y Aguaded-Gómez, J. I. (2015). Alfabetización comunicativa y competencia mediática en la sociedad hipercomunicada. Revista Digital de Investigación en Docencia Universitaria, 9(1), 37-56.

DOI: https://doi.org/10.19083/ridu.9.379

Chibás, F. (2012). Educomunicação na gestão educacional criativa em projetos corporativos EAD: um estudo de caso. Hermes, 6, 77-97.

DOI: https://doi.org/10.21710/rch.v6i0.120

Cobo, C., y Moravec, J. W. (2011). Aprendizaje invisible. Hacia una nueva ecología de la educación. Barcelona, España: Editorial de la Universitat de Barcelona.

Comisión Europea (2008). Education, Youth and Culture. Recuperado de http://europa.eu/rapid/press-release_PRES-08-120_en.htm

Davies, L. (2005). Schools and war: Urgent agendas for comparative and international education. Compare: A Journal of Comparative and International Education, 35(4), 357-371. DOI: https://doi.org/10.1080/03057920500331561

Vivat Academia. Revista de Comunicación. 15 septiembre 2019 /15 diciembre 2019, n 148, 23-40 
Caldeiro Pedreira, M.; Torres-Toukoumidis, A., Romero-Rodríguez, L. M. y Aguaded, I. Noción de la educomunicación en las organizaciones intergubernamentales

Freire, P. (1999). La importancia de leer y el proceso de liberación. Buenos Aires, Argentina: Siglo XXI.

García Matilla, A. (2002). Educomunicación en el siglo XXI. Recuperado de http://www2.uned.es/ntedu/asignatu/7_Agutin_G_MatillaI1.html

Gozálvez, V. (2013). Ciudadanía mediática: una mirada educativa. Madrid, España: Dykinson.

Martín, A. G. (2007). Integración curricular de las TIC y educación para los medios en la sociedad del conocimiento. Revista Iberoamericana de educación, 45, 141-156.

Heinmiller, B. T. (2007). Do intergovernmental institutions matter? The case of water diversion regulation in the Great Lakes Basin. Governance, 20(4), 655-674. DOI: https://doi.org/10.1111/j.1468-0491.2007.00376.x

Kaplún, M. (1997). De medios y fines en comunicación educativa. Chasqui. Revista Latinoamericana de Comunicación, (58).

DOI: https://doi.org/10.16921/chasqui.v0i4.2801

LeCompte, M. D., y Schensul, J. J. (2012). Analysis and interpretation of ethnographic data: A mixed methods approach. Maryland, EEUU: Rowman Altamira.

Tornero, J. M. P., y Varis, T. (2012). Alfabetización mediática y nuevo humanismo. Barcelona, España: Editorial Uoc.

Ralón, L., Vieta, M., y Vásquez de Prada, M. L. (2004). (De) formación en línea: acerca de las desventajas de la educación virtual. Comunicar, (22).

Romero-Rodríguez, L. M., de-Casas-Moreno, P., y Torres-Toukoumidis, Á. (2016). Dimensiones e indicadores de la calidad informativa en los medios digitales. Comunicar, 24(49), 91-100. DOI: https://doi.org/10.3916/c49-2016-09

Saini, M., \& Shlonsky, A. (2012). Systematic synthesis of qualitative research. Nueva York, EEUU: OUP USA.

Hernández Sampieri, R.; Fernández Collado, C., y Baptista Lucio, P. (2006). Metodología de la investigación. México: McGraw-Hill.

Sánchez, J., y Sandoval, Y. (2012). Claves para reconocer los niveles de lectura crítica audiovisual en el niño. Comunicar, 19(38), 1130-120.

DOI: https://doi.org/10.3916/c38-2012-03-02

Vivat Academia. Revista de Comunicación. 15 septiembre 2019 /15 diciembre 2019, n 148, 23-40 
Caldeiro Pedreira, M.; Torres-Toukoumidis, A., Romero-Rodríguez, L. M. y Aguaded, I. Noción de la educomunicación en las organizaciones intergubernamentales

Serrano, M. J. H., Arellano, P. R., Graham, G., y Greenhill, A. (2017). Del prosumidor al prodiseñador: el consumo participativo de noticias. Comunicar: Revista científica iberoamericana de comunicación y educación, 50, 77-88.

DOI: https://doi.org/10.3916/c50-2017-07

Scolari, C. A. (2015). Los ecos de McLuhan: ecología de los medios, semiótica e interfaces. Palabra Clave, 18(4), 1025-1056.

DOI: https://doi.org/10.5294/pacla.2015.18.4.4

Sterling, S., y Huckle, J. (2014). Education for sustainability. Berkshire, Inglaterra: Routledge.

Tyner, K. (2008). Audiencias, intertextualidad y nueva alfabetización en medios. Comunicar: Revista científica iberoamericana de comunicación y educación, 30, 79-85. DOI: https://doi.org/10.3916/c30-2008-01-012

UNESCO. (1982). The Grünwald Declaration on Media Education. Grünwald Alemania: Editorial UNESCO.

UNESCO. (1991). Media Education in the ASEAN Countries. Jakarta, Indonesia: Editorial UNESCO.

Uribe Tirado, A. (2010). La alfabetización informacional en Iberoamérica. IBERSID, 165176.

\section{AUTORES}

\section{Mari Carmen Caldeiro Pedreira}

Doctora en Comunicación y Educación por la Universidad de Huelva (España). Máster en Formación del Profesorado por la Universidad de Santiago de Compostela (España). En la actualidad profesora del Departamento de Pedagogía y Didáctica de la Universidad de Santiago de Compostela (España).

mcarmen.caldeiro@usc.es

Orcid ID: http:// orcid.org/0000-0003-0160-3682

ResearchGate: https://www.researchgate.net/profile/Mari_Pedreira

\section{Ángel Torres Toukoumidis}

Doctor en el programa de Doctorado Interuniversitario en Comunicación bajo la línea de investigación «Educomunicación y Media Literacy» (2014), de las universidades de Huelva, Sevilla, Málaga y Cádiz. Realiza una investigación sobre la Gamificación como estrategia integral de evaluación de políticas públicas y educación ciudadana. Máster en Comunicación con Fines Sociales por la Universidad de Valladolid, licenciado en

Vivat Academia. Revista de Comunicación. 15 septiembre 2019 /15 diciembre 2019, n 148, 23-40 
Caldeiro Pedreira, M.; Torres-Toukoumidis, A., Romero-Rodríguez, L. M. y Aguaded, I. Noción de la educomunicación en las organizaciones intergubernamentales

Estudios Liberales por la Universidad Metropolitana (Venezuela) homologado en Ciencias Políticas por la Universidad Complutense de Madrid. Revisor Científico de Comunicar, Revista Científica de Educación y Comunicación. Estancia Internacional de investigación y docencia (seis meses) en la Universidad de Gotemburgo (Suecia). Investigador asociado del grupo MUL (Nya Media, Undervisning och Lärande- Nuevos medios, Enseñanza y Aprendizaje) y del Laboratorio de diseño de Juegos del Spelvetenskapliga kollegiet de Gotemburgo. Grupo de Comunicaciones para la red de investigación Alfamed. Actualmente docente de la Universidad Politécnica Salesiana, Ecuador en la facultad de Comunicación Social.

atorrest@ups.edu.ec

Orcid ID: http://orcid.org/0000-0002-7727-3985

ResearchGate: https://www.researchgate.net/profile/Angel_Torres-Toukoumidis

\section{Luis M. Romero Rodríguez}

Doctor en Comunicación por la Universidad de Huelva (España), Máster en Comunicación Social por la Universidad de Almería (España) y Especialista en Derecho y Políticas Internacionales por la Universidad Central de Venezuela y en Docencia Universitaria por la Universidad Nueva Esparta (Venezuela). Docente de las Universidades Internacional de La Rioja (España), Nebrija (España), Internacional de Andalucía (España), Huelva (España) y de la Universidad de Cádiz (España). Investigador especializado en crítica de medios de comunicación, competencias mediáticas y gamificación. Editor adjunto de la Revista Comunicar (España) y editor jefe

de la Revista Retos (UPS-Ecuador). Miembro del Grupo Comunicar y del Grupo Ágora (PAI-HUM-648) de la Universidad de Huelva.

luis.romero@urjc.es

Orcid ID: http:/ / orcid.org/0000-0003-3924-1517

ResearchGate: https://www.researchgate.net/profile/Luis_Romero-Rodriguez2

\section{Ignacio Aguaded}

Catedrático de Universidad de Educación y Comunicación en la Universidad de Huelva. Preside el Grupo Comunicar, colectivo veterano en España en «Media Literacy». Director del Grupo de Investigación «Ágora» dentro del Plan Andaluz de Investigación (HUM-648). Director del Máster Internacional de Comunicación y Educación (UNIA/UHU) y coordinador UHU del Programa Interuniversitario de Doctorado en Comunicación (US, UMA, UCA, UHU).

ignacio@aguaded.es

Orcid ID: http:// orcid.org/0000-0002-0229-1118

ResearchGate: https://www.researchgate.net/profile/Ignacio_Aguaded

Vivat Academia. Revista de Comunicación. 15 septiembre 2019 /15 diciembre 2019, n 148, 23-40 